\title{
Subsurface Cavity Detection Using Electrical Resistivity Tomography (Ert); A Case Study from Southern Quetta, Pakistan
}

\section{Syed Ali Abbas, Muhammad Saeed, Mukhtiar Ghani*, Taseer Ahmad}

Geological Survey of Pakistan, HQ, Sariab Road Quetta, Office No.14, Block No.07, Geological Survey of Pakistan, Sariab Road, Quetta, Pakistan. *Corresponding Author Email: mukhtiarghani@uop.edu.pk

\section{DOI: 10.2478/pjg-2020-0012}

\section{Abstract:}

Dipole-dipole electrical resistivity tomographic method was applied to investigate the subsurface cavities at Staff Welfare Hospital \& School Quetta. A total of 890-meter profile line was covered along five smaller profile lines and fracture zones with maximum 21 meters interval. The cavity system along profile line- 1 and 2 was very restricted and had no direct impact on infrastructure while major cavity beneath the building was traced at profile line- 3 and line- 4 thus constituting a $\sim 20 \mathrm{~m}$ wide cavity system with 3-4 small interconnected cavities between depths of 7 to 21 meters. This system was also traced at profile line- 4 at a depth of 10 meters having a reduced width of $10 \mathrm{~m}$. At profile line-5, a few other cavities were detected that proved imperceptible due to limitations in data acquisition. To conclude, the cavity systems traced in profile line-3 and profile line- 4 were the most perilous ones and are commonly the foremost reason for building collapse.

Keywords: Natural disaster; Quetta Valley; Geophysical Techniques.

\subsection{Introduction:}

Quetta is the provincial capital of Balochistan province surrounded by mountain ranges of Chiltan in west, Murdar Ghar in east and Zarghoon to the south [1]. The valley is buried due to tectonic depression with karstic bedrock architecture, surrounded by Jurassic and cretaceous ranges with quaternary sediments composing the basin fill deposites [2-4]. Subsidence and land collapsing are a common phenomenon in Quetta city [5]. Abandoned kareez network (subsurface canals) is blamed to cause majority of them [6]. Due to these many fissures are being developed in the specially in the middle parts of the city, where the subsurface is mostly consists of thick loos material. The fissures zones are as much as 1000 meters long 200 meters wide and individually, 3 meters deep and 1 meter wide at often damages many buildings. The fissure are direct responses to underground cavity collapse and compaction of sediments with groundwater withdrawal. The city is home to a large population are fully all supported on this venerable platform. To find our overall subsidence, an overall GPS network is installed which suggest a continuous subsidence in the valley. It is important to study subsurface for probable cavities and take precautionary measure before any natural disaster. For cavity detection and venerable places, geophysical techniques are most favorable option [5].

Electrical Resistivity Tomography (ERT) is one of the most employed technique in geophysics for geomorphological research [7]. ERT is mainly used for issues concerning deformation of slope and accompanying landform stabilities [8]. Extrapolation of geomorphic and speleological investigations have been used to obtain significant results in elevated and gravitationally spread ridges having crevice-type fractures/caves [9]. Resistivity survey is a new insight to look into the internal structure of disrupted subsurface [9]. High resolution 2D resistivity images are useful in interpretation of shallow structures (ranging from a few meters to about one hundred meters deep). The images obtained from this method complements the one obtained from more traditional method of Vertical Electrical Sounding (VES) technique. VES mainly aims at determining the depths of horizontal 1D structures from the surface up till several hundred meters depth [10]. Resistivity measurements can stimulate direct response of lithological and hydrological elements of subsurface. The depth of investigation varies with separation of potential and current electrodes in the survey [11]. Resistivity values are generally affected by water saturation and connectivity of pore spaces. The air-filled voids will give high resistivity values as compared to water filled ones [12]. Electrical method is inexpensive, fast and non-invasive technique for obtaining useful subsurface information [13]. In this study, electrical resistivity tomographic study of staff welfare hospital (Figure 1.) was conducted to model subsurface cavities and analyze risk to building collapse.

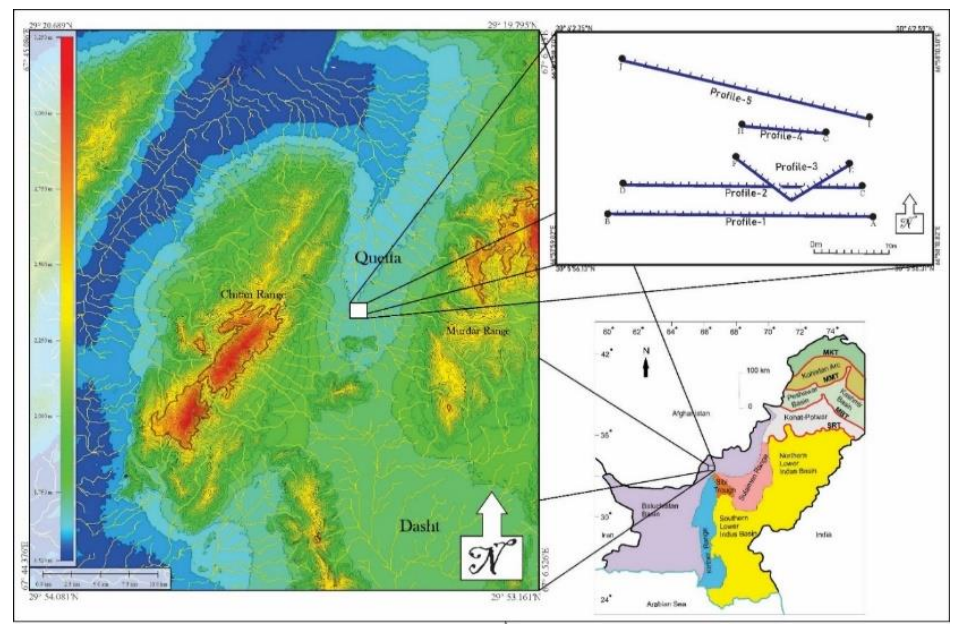

Figure 1: Location map of the research area with regional map of Quetta and Pakistan.

\subsection{Material and Methods}

To investigate the possibility of subsurface cavities beneath staff welfare hospital, the dipole-dipole electrical resistivity tomography method was employed. ERT data along five profiles were acquired using ABEM SAS-300 Terrameter for various electrode spacings (Table 1) to inspect the depths of 28 and 40 meters. Detailed description regarding acquisition parameters are given below in the Table 1. 
Table 1: Acquisition parameters for ERT survey

\begin{tabular}{|c|c|c|c|c|c|}
\hline Profile & Start & End & $\begin{array}{l}\text { Electrode } \\
\text { Spacing }\end{array}$ & $\begin{array}{l}\text { Length } \\
\text { meters }\end{array}$ & Orientation \\
\hline Line-1 & 300557.4 / 66588.8 & 300557.4 / 66580.5 & 7 meters & 220 & East-West \\
\hline Line-2 & $300558 / 66588.5$ & 300558.3 / 66581.0 & 7 meters & 213 & East-West \\
\hline Line-3 & $300558.9 / 665808.1$ & 300559.0 / 665804.5 & 7 meters & 120 & East-West \\
\hline Line-4 & $300559.7 / 665807.3$ & 300559.8 / 665804.7 & 7 meters & 85 & East-West \\
\hline Line-5 & $300600.6 / 665809$ & 300601.6 / 66580.8 & 10 meters & 200 & East-West \\
\hline
\end{tabular}

The general resistivity, $\rho$ s formula is given as, $\rho s=\mathrm{V} / \mathrm{I} \times \mathrm{A} / \mathrm{L}$. where $\mathrm{V} / \mathrm{I}$ is resistance, $\mathrm{R}$ calculated by ration of potential difference, $\mathrm{V}$ current flow, $\mathrm{I}$. The $\mathrm{A} / \mathrm{L}$ is the Geometric Factor, $\mathrm{K}$ can have different value for different survey configuration. In current survey, a dipole-dipole configuration is adopted with is given in detail below. Electrical Resistivity Soundings, ERS is a geophysical method used for underground resistivity imaging [12]. For electrical profiling of Constant Separation Traversing (CST), collinear array configuration was adapted for determining lateral resistivity variations at fix depths. The current and potential electrodes were moved forward along a profile line with constant spacing between electrodes. There are two array types for CST configuration i.e., dipole-dipole and pole-dipole arrays.

Every configuration of electrodes comes with its benefits and limitations for subsurface resistivity modeling. Each configuration consists of current input electrodes (AB) and potential electrodes (MN). In dipole-dipole method, the current electrodes (AB) are placed on one side with a spacing "a" while potential electrodes (MN) are placed with same spacing "a" at a distance "na" from AB. Measurements are taken as MN electrode moves across different values of $n$ thus generating a profile beneath the surface. A generalized configuration of dipole-dipole arrays is shown in fig 2 demonstrating electric field lines and resultant equipotential surfaces. After completing an array with movement of $\mathrm{MN}$ electrode, the $\mathrm{AB}$ electrodes are displaced on. Dipole-dipole array configuration is common for covering large spaces owing to the convenient logistics on field [14]. The dipole-dipole array measures the curvature of the potential difference and therefore, is the most sensitive method to note subtle resistivity changes between the electrodes. The best thing in dipole-dipole configuration is that both current (A\&B) and potential (M\&N) electrode spacing are same i.e. "a" and this spacing is an integral multiple ' $n$ ' of "a" i.e. "na".

The apparent resistivity is calculated by the formula:

\section{$\rho \mathbf{a}=\mathbf{n}(\mathbf{n}+1)(\mathrm{n}+2) \pi \mathrm{a} \Delta \mathrm{V} / \mathrm{I}$}

Where

$\rho \mathrm{a}=$ apparent resistivity $(\mathrm{Ohm}-\mathrm{m})$

$\mathrm{n}=$ dipole number

$\mathrm{a}=$ dipole spacing (meters)

$\Delta \mathrm{V}=$ potential difference (milli Volts)

$\mathrm{I}=$ Current (milli amperes)

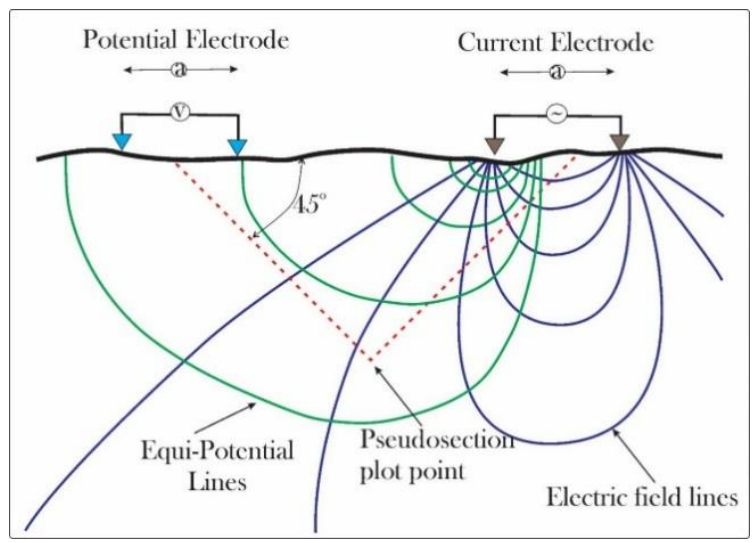

Figure 2: Graphical illustration of Dipole-Dipole configuration.

\subsection{Interpretation and Results:}

\subsection{Profile Line-1 (AB):}

The apparent resistivity along profile line-1 is represented by pseudo section (Figure 3A.) and 3D model (Figure 4A.). This section shows a uniform distribution of apparent resistivity $(<20 \Omega \mathrm{m})$ depicting a uniform material (Figure 3A.). Between arrays 2 and 5 , a shallow medium resistivity $(20-26 \Omega \mathrm{m})$ closure is observed that shows small-interconnected cavities. The chain is 10-meter-long and 7 meter wide. Another moderate resistivity point about 7 to 9 meters thick is present between Array 8 and 10 at the depth of 17 meters. The closure shows a medium to high resistivity of $>28 \Omega \mathrm{m}$, representing another cavity system. 3D representation of apparent resistivity illustrates that there are two interconnected cavities ( $\mathrm{C} 1$ and $\mathrm{C} 2$ ) at first resistivity closure and two (C3 and C4) at second closure (Figure 4A.).

\subsection{Profile Line-2 (CD)}

The pseudo section in figure 3B embodies the apparent resistivity along profile line- 2 and its 3D illustration in fig 4B. The apparent resistivity ranges from 2 to $30 \mathrm{ohm}$-meters. There is a moderate resistivity anomaly at the start of profile with peak values of $28 \mathrm{ohm}$-meters while middle part of the section exhibits regular smooth behavior (Figure 3B.). A high resistivity point ( $>25 \Omega \mathrm{m})$ in the start of profile (C5) is indicating some loose material in shallow subsurface (Figure 4B). In array-7, C6 point is a small closure in fig 2B. depicting a cavity of 1 meter diameter. Another relative moderate apparent resistivity 
$(\sim 25 \Omega \mathrm{m})$ closure is there between Array $17-22$ at a depth of 17 meters that may be a small cavity (C6) surrounded by some loose material. In 3D representation, a constant apparent resistivity layer is set at $20 \Omega \mathrm{m}$ and these three points show anomalous resistivity above constant line (Figure 4B.).

\subsection{Profile Line-3 (EF)}

Figure $3 \mathrm{C}$ is the resistivity cross section along E-W oriented $120 \mathrm{~m}$ long profile. Low values of apparent resistivity $(<30 \Omega \mathrm{m})$ are observed throughout the profile. Similar resistivity pattern indicates the absence of large sized cavities. However, high resistivity zone ( $>80 \Omega \mathrm{m}) 80-100 \mathrm{~m}$ deep along profile clearly defines a cavity. This cavity can be inferred as localized but poses a potential threat to the infrastructure.

\subsection{Profile Line-4 (GH)}

The apparent resistivity distribution along profile line- 4 is represented by pseudo section in figure 3D. Generally ranging from 2 to $45 \Omega \mathrm{m}$ throughout the profile, however there is a high resistivity anomaly present between Array 4 and 6 with peak values of $40-45 \Omega \mathrm{m}$ (Figure 3D.). This very zone looks like the continuation of previously observed high resistivity value zone in Profile line-3 and this trend is continuous from profile line-3 to 4 . The zone seems to close 7-10 meters deep and it may very much be the end of that cavity. Two major cavity systems can be seen at the advent (C12) and mid (C13) of the profile line (Figure 4D.).

\subsection{Profile Line-5 (IJ)}

Resistivity survey along profile line-5 was conducted with 10-meter dipole spacing to inspect the extent of exposed cavity at higher depth. Figure 3E. is the pseudo section along this profile line gauging general apparent resistivity values between 2 and $105 \Omega \mathrm{m}$. There is a high resistivity anomaly at the center of the profile between array 10 and 12 spiking peak values of $90-105 \Omega \mathrm{m}$. There are two more medium resistivity zones detected on this profile. First, at array 7 gives medium resistivity values of 35-40 $\Omega \mathrm{m}$ at 15-meter depth extending down till 25 meters. Second, at array 17 also gives similar response about 12 meters deep. Along this profile line-5, this is the main cavity system indicated by C14, C15 and C16 in 3D resistivity model (Figure 4E.).

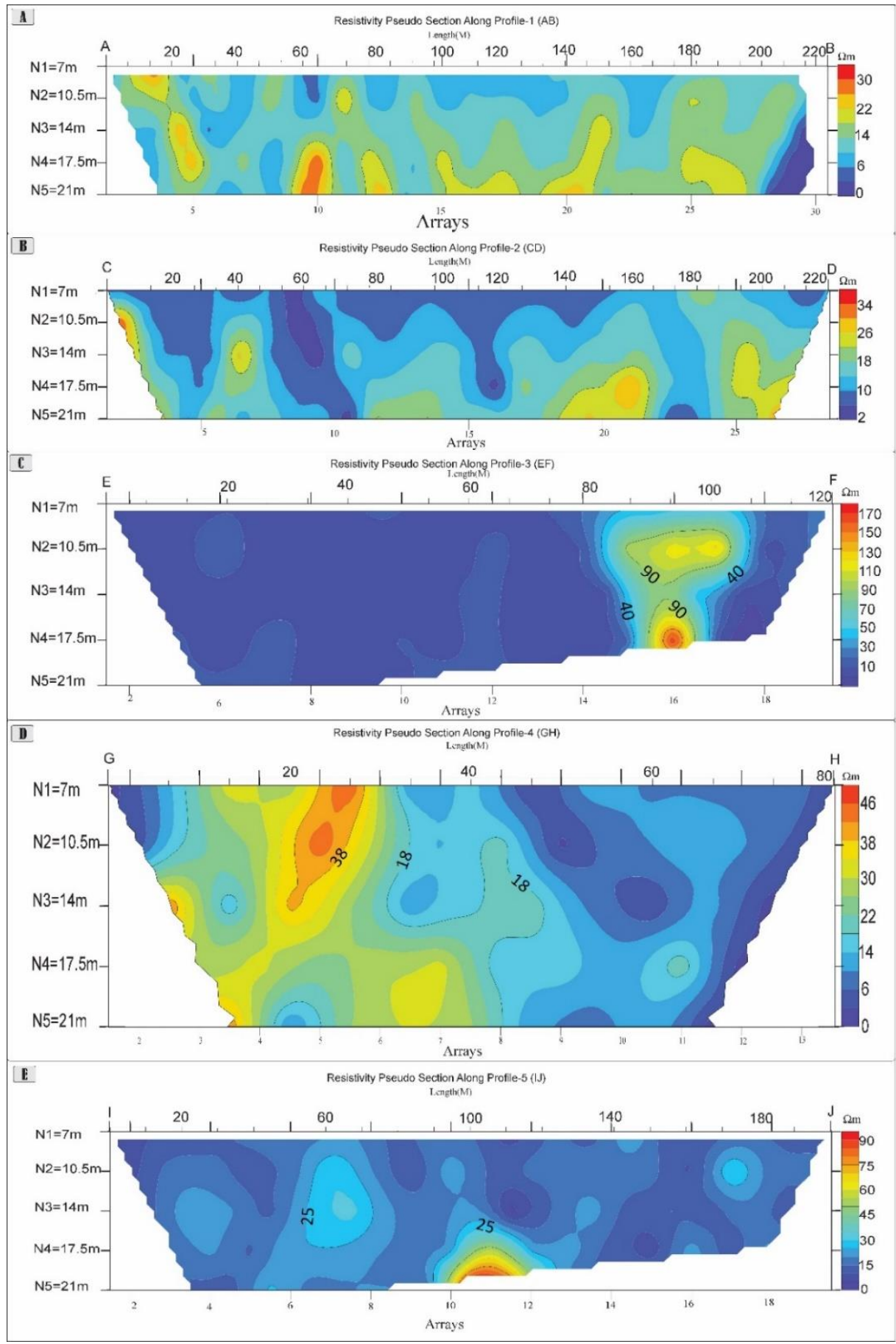


Figure 3: Apparent Resistivity Pseudo Section along Profile-1 to Profile-5.
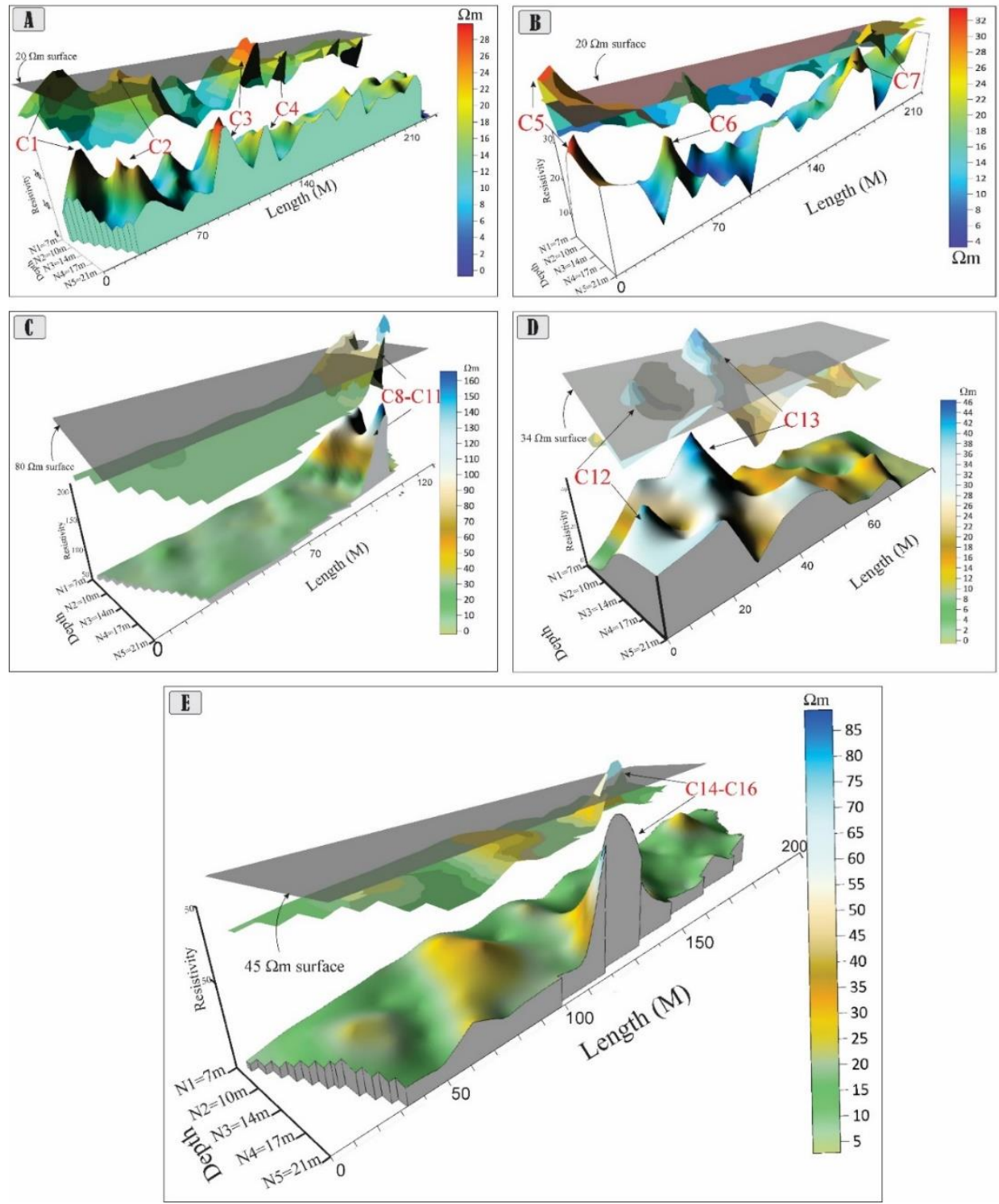

Figure 4: Apparent Resistivity 3D Pseudo Section along Profile-1 to Profile-5 with representation of cavities modeling.

\subsection{Recommendation}

There are total of 17 cavities present in the study area of which consist of 5 set of interconnected cavity systems (Figure 5.). The hazardous cavities are concentrated in few spots and cut out through the main building. The building in erected on an uninvestigated subsurface which is posing risk to the building sustainability. These individual cavities are mostly smaller i.e. $<5$ meters with depth of $>20$ meters and the cavities are sur rounded by loos material. The building may not collapse suddenly but posing little chances of sustainability.

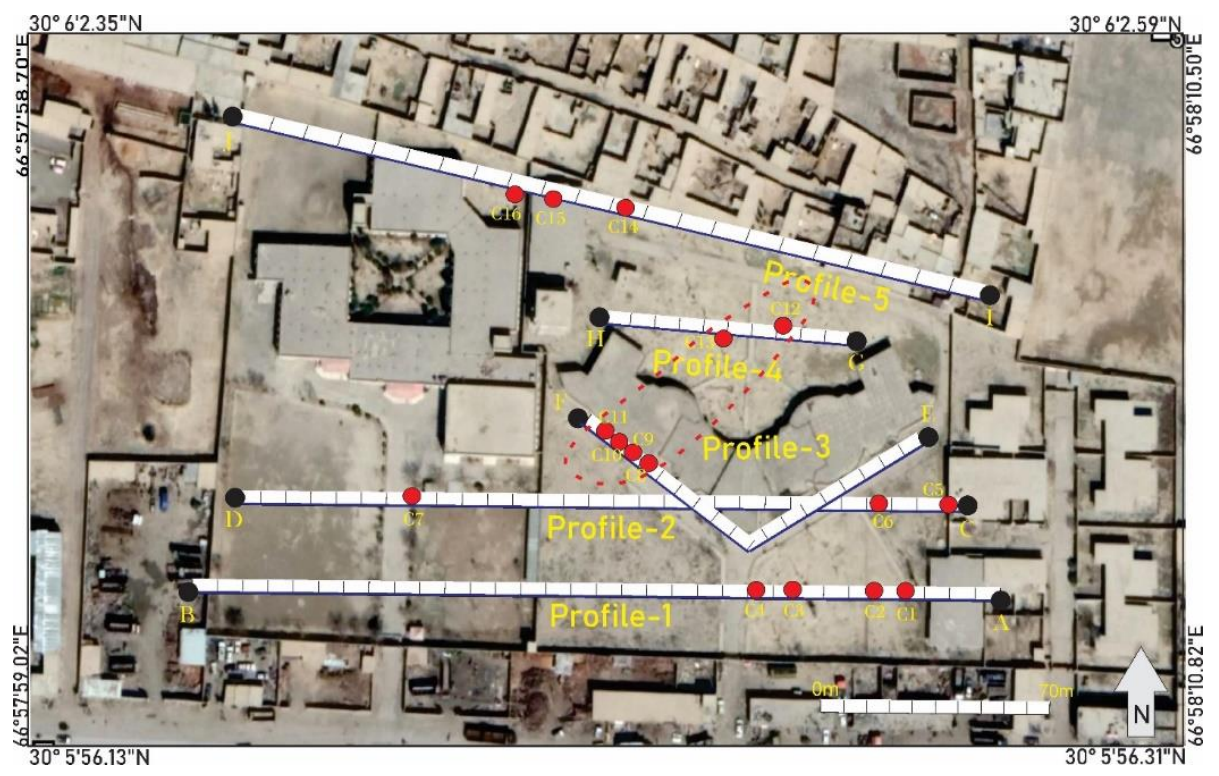


Figure 5: Location map of the research area with location of cavities on profile lines.

\subsection{Conclusion}

Based on the observations from total profile data of the project area, it is interpreted that significant cavities are present directly beneath the main entrance of hospital building. A series of cavities are present from surface to the depth of 21 meters. Along profile line -3, several cavities are present beneath array14 to array-18. (Figure 3C.; Figure 4C.). Same cavity are encountered at profile line- 4 between array-3 and array-6 at seven (7) meters depth (Figure 3D.; Figure 4D.). The ground beneath the hospital location on both profiles is dominated by medium to high resistivity that indicates water-saturated or open cavity. The model suggests that this cavity system passes under the main building and consequently make it venerable to subsidence. In addition, the model suggests that cavity system is shallower in the northern section and goes deeper southwards beneath the main infrastructure.

\subsection{Acknowledgement}

Geological Survey of Pakistan, Quetta are highly acknowledged for financial, logistic and instrumental support for these studies.

\subsection{References}

[1] Z. Kakar, S.M. Shah, and M.A. Khan, "Scarcity of water resources in rural area of Quetta District; challenges and preparedness", In: IOP Conference Series: Materials Science and Engineering, Vol 1. IOP Publishing, Pp. 12-13, 2018.

[2] K. Murray, Z. Sagintayev, M. Sultan, S. Khan, D.B. Becker, and R. Becker, "An integrated approach for the assessment and development of renewable groundwater resources in the Quetta valley, Pakistan", In: Geological Society of America Abstracts with Programs, Vol 6., Pp. $154,2007$.

[3] B. Muhammad, S. Khan, M. Mohibullah, A.K. Kasi, and M. Ghani, “Planktonic foraminiferal biostratigraphy and depositional setting of the Cretaceous Parh Limestone, Quetta, western Sulaiman Fold-Thrust Belt, Balochistan, Pakistan”, Journal of Himalayan Earth Sciences, 51, Pp. 8-23, 2019.

[4] I.H. Durrani, S. Adnan, M. Ahmad, S. Khair, and E. Kakar, "Observed long-term climatic variability and its impacts on the ground water level of Quetta alluvial”, Iranian Journal of Science and Technology, Transactions A: Science, Vol. 42, Pp. 589-600, 2018.

[5] A.S. Khan, S.D. Khan, and D.M. Kakar, "Land subsidence and declining water resources in Quetta Valley, Pakistan", Environmental Earth Sciences, Vol. 70, Pp. 2719-2727, 2013.

[6] J. Huang, K. Shuhab, A. Wulamu, W. Crupa, A. Khan, D. Kakar, Kasi, “A Surface Deformation in Quetta Valley, Balochistan, Pakistan”, In: AGU Fall Meeting Abstracts, 2015.

[7] T. Günther, and C. Rücker, “Electrical Resistivity Tomography (ERT) in geophysical applications-state of the art and future challenges”, In: Schlumberger Symposium--100 years of electrical imaging, Paris, (January), 2012.

[8] N. Greggio, B. Giambastiani, E. Balugani, C. Amaini, and M. Antonellini, "High-resolution electrical resistivity tomography (ERT) to characterize the spatial extension of freshwater lenses in a salinized coastal aquifer", Water, Vol. 10, Pp. 1067, 2018.

[9] J.E. Chambers, O. Kuras, P.I. Meldrum, R.D. Ogilvy, and J. Hollands, "Electrical resistivity tomography applied to geologic, hydrogeologic, and engineering investigations at a former waste-disposal site, Geophysics, Vol. 71, Pp. B231-B239, 2006.

[10] B. Zhou, "Electrical Resistivity Tomography: A Subsurface-Imaging Technique. In: Applied Geophysics with Case Studies on Environmental, Exploration and Engineering Geophysics. Intech Open, 2018.

[11] W. Lowrie, "Fundamental of Geophysics", Cambridge University Press, Pp. 354, 1997.

[12] A.S. Ogungbe, J.A. Olowofela, O.J., Da-Silva, A.A. Alabi, and E.O. Onori, "Subsurface characterization using electrical resistivity (Dipole-Dipole) method at Lagos State University (LASU) Foundation School, Badagry”, Advances in Applied Science Research, Vol. 1, Pp. 174-181, 2010.

[13] A.A. Zohdy, “A new method for differential resistivity sounding”, Geophysics, Vol. 34, Pp. 924-943, 1969.

[14] M. Blome, H. Maurer, and K. Schmidt, "Advances in three-dimensional geoelectric forward solver techniques", Geophysical Journal International, Vol 176, Pp. 740-752, 2009. 